\title{
Favorable Effect of Bromocriptine and Pentoxifylline in Mild Graves Disease Orbitopathy: A Randomized, Open, Clinical Trial
}

Paloma Almeda-Valdés ${ }^{1}$, Daniel Cuevas-Ramos ${ }^{1}$, Miguel A. Gómez-Sámano ${ }^{1}$, Francisco Cárdenas-Velázquez ${ }^{2 *}$, Bernard Pérez-Enríquez ${ }^{1}$, Arturo Abundes Corona ${ }^{1}$, and Francisco J. Gómez-Pérez ${ }^{1}$

${ }^{1}$ Instituto Nacional de Ciencias Médicas y Nutrición Salvador Zubirán, México City. Department of Endocrinology and Metabolism

${ }^{2}$ Instituto Nacional de Ciencias Médicas y Nutrición Salvador Zubirán, México City. Department of Ophthalmology.

Received: April 28, 2017;Accepted: May 20, 2017; Published: June 20, 2017

*Corresponding author: Dr. Francisco J. Gómez-Pérez, MD. NInstituto Nacional de Ciencias Médicas y Nutrición Salvador Zubirán, Endocrinology and Metabolism Department. Vasco de Quiroga No. 15. Colonia Belisario Dominguez Sección XVI. Tlalpan. Ciudad de México., México. 14080. Tel:+5255133891;Fax: +555513-3891;E-mail: gomezperezfco@gmail.com

\begin{abstract}
Background

Graves' orbitopathy (GO) is the most frequent extra thyroidal manifestation of Graves' disease. Treatment for mild cases consists in euthyroidism restoration and topical treatments. Aim: To evaluate the effect of bromocriptine (BRC) or pentoxifylline (PTX) added to methimazole (MTZ) in proptosis, clinical activity, and quality of life in individuals with mild GO. Methods: Randomized, open, clinical trial. Forty-five patients with mild GO with an onset of less than one year were randomly assigned to MTZ, MTZ+BRC, or MTZ+PTX treatment during one year. Proptosis was measured with exophthalmometry, clinical activity was evaluated using the Clinical Activity Score (CAS), and the Graves' Orbitopathy Quality Of Life (GO-QOL) questionnaire was also applied. Results: Thirty-five patients were analyzed, 13 assigned to MTZ, 9 to MTZ+BRC, and 13 to MTZ+PTX. After one year, a decrease in proptosis was documented in the MTZ+BRC group. Proptosis on right eye (OD) decreased from 20 [18-21] to 18 [16-18] mm ( $\mathrm{p}=0.006)$, and on left eye (OS) from 20 [19-20] to 18 [16-18] $(\mathrm{p}=0.006)$. MTZ+PTX treatment also decreased proptosis. In OD from 19 [17.5-19] to $16.5 \mathrm{~mm}$ [16-19] $(\mathrm{p}=0.120)$ and in OS from 19 [17.5-19] to 17 [16-20] $(\mathrm{p}=0.025)$. No change was observed on the MTZ group. The CAS and GO-QOL scores significantly improved in all groups. Conclusions: BRC or PTX treatments added to MTZ improved proptosis in individuals with recent onset, mild GO. CAS and quality of life similarly improved in all groups.
\end{abstract}

\section{Clinical Trial Registration Number: NCT01893450}

Key words: Hyperthyroidism; Graves' ophthalmopathy; exophthalmos; bromocriptine; pentoxifylline

\section{Introduction}

Graves' orbitopathy (GO) is the most frequent extra thyroidal manifestation of Graves' disease. It has an annual adjusted incidence of 16 women and 2.9 men per 100,000 [1] and an estimated prevalence between 0.1 and $0.3 \%$ [2]. In most cases GO is mild; however, in 3 to $5 \%$ of the cases it has a severe presentation [3].
At present, available treatments are insufficient [4] and are focused on identifying patients with sight-threatening GO whom require aggressive intervention with glucocorticoids, orbital decompression, and/or radiotherapy. Patients with moderate to severe GO defined with lid retraction $>2 \mathrm{~mm}$, moderate or severe soft tissue involvement, exophthalmos $>3$ $\mathrm{mm}$ above normal, inconstant or constant diplopia, also qualify for immunosuppressive therapy [3].

For mild cases, management is restricted to hyperthyroidism correction and topical treatments [3]. However, patients with mild GO have a reduced quality of life [5] and $13.5 \%$ progress to moderate or severe manifestations without treatment [6]. Some studies evaluating agents for mild GO have shown promising results. Long-acting release octreotide showed a significant reduction in proptosis compared to placebo [7]. Colchicine demonstrated an amelioration of the clinical activity score (CAS) and improvement in the signal intensity ratio of the recti muscle in magnetic resonance imaging [8]. Allopurinol plus nicotinamide was associated with a decrease in soft tissue inflammation [9]. Diclofenac showed a significant reduction in the muscle restriction score and CAS [10]. A multicenter, randomized clinical trial showed utility of selenium improving quality of life, eye involvement, and reducing GO progression [11]. A small study using pentoxifylline (PTX) improved GO severity compared to placebo [12]. Lastly, case reports and small case series have shown improvement in GO activity with bromocriptine (BRC) [13-15]. The postulated mechanisms explaining the favorable effect of BRC are the inhibition of immune cells and dropping of TSH secretion [13].

Besides the well-known roles of prolactin (PRL) in reproduction and lactation, it also functions as a cytokine implicated in the immune response [16]. Lymphocytes and other immune cells express both PRL and the PRL receptor. PRL functions as a local regulator of the lymphocyte proliferation $[17,18]$, increases cytokine production [19] including interferon gamma $[20,21]$, among other immunological effects. Modulation 
of PRL may have an effect in the onset and progression of autoimmune diseases [22].

The primary aim of this study is to evaluate the effect of BRC and PTX added to methimazole (MTZ) on the proptosis and CAS in patients with mild GO, and secondarily to assess their effect on quality of life.

\section{Materials and Methods}

\section{Subjects}

From February 2009 through July 2013, we recruited patients between 18 and 60 years-old with mild GO of less than 12 months onset. Mild GO was defined as patients whose features have only a minor impact on daily life, insufficient to justify immunosuppressive or surgical treatment. They had minor lid retraction $(<2 \mathrm{~mm})$, mild soft tissue involvement, exophthalmos $<$ $3 \mathrm{~mm}$ above normal, transient or no diplopia, and corneal exposure responsive to lubricants [3]. Exclusion criteria included previous treatment for GO, current tobacco use, concomitant illnesses such as diabetes, uncontrolled hypertension, cardiovascular disease, asthma, chronic kidney disease, hematologic diseases, gastrointestinal bleeding, use of immunosuppressant treatment (e. Gr. Steroids), or other ophthalmological diseases. Elimination criteria included severe adverse events related to treatment, and $<80 \%$ treatment adherence.

\section{Experimental Design}

This was a randomized, open, clinical trial. Patients were assigned using a random number table with sealed envelopes to receive treatment with either: 1) MTZ $30 \mathrm{mg}$ daily, 2) MTZ $30 \mathrm{mg}$ daily plus PTX $400 \mathrm{mg}$ twice daily, or 3) MTZ $30 \mathrm{mg}$ daily plus BRC $2.5 \mathrm{mg}$ twice daily. To minimize side effects and improve tolerance, PTX and BRC were titrated over a two-week period. Throughout the study, a block-replace regimen was used adding levothyroxine in order to maintain euthyroidism [16].

Patients attended for a monthly evaluation for the first four months, and thereafter every two months until the study ended. Each subject completed 10 visits over one year. A Hertel exophtalmometer was used to evaluate exophthalmos. Proptosis, in millimeters, was recorded in right eye (OD) and left eye (OS). The CAS was calculated as the sum of the following items: spontaneous retrobulbar pain, pain on attempted eye movements (upward, side-to-side, and downward gazes), conjunctival redness, redness of the eyelids, chemosis, swelling of the caruncle, and swelling of the eyelid [23]. Evaluations were performed by three trained and standardized Endocrinologists that were blinded for the assigned treatment. Concordance between Endocrinologists in proptosis measurements compared to an expert Ophthalmologist were satisfactory (kappa $=0.94, \mathrm{p}<$ 0.001).

We evaluated the quality of life at baseline and at the end of the study with the Graves' Orbitopathy Quality Of Life (GOQOL) questionnaire [24]. The questionnaire was not available in Spanish; therefore it was translated to Spanish and then translated back to English by a native English speaker to assure accuracy. The Spanish version is now available on the European Group of Graves' Orbitopathy (EUGOGO) webpage: http://www. eugogo.eu/_downloads/clincial_evaluation/GO_QOL_ES.pdf. The questionnaire evaluates visual functioning and appearance. A score of 1, 2, or 3 is assigned to each question indicating the limitation as severe, mild, or absent, respectively. The scores are added to obtain a raw score and the final score is calculated as follows: (raw score -8 ) $/ 16 * 100$. The final score ranges from a minimum of 0 (full limitation) to 100 (no limitation). An increase in the score indicates improvement and a decrease indicates worsening.

Blood samples were obtained to assess thyroid function. Side effects of medications were recorded at follow-up visits. Adherence to treatment was evaluated by pill count. The primary outcomes were the change in proptosis and in the CAS.

The study was approved by the Comité de Ética en Investigación del Instituto Nacional de Ciencias Médicas y Nutrición Salvador Zubirán (\#1950). Written informed consent was obtained from all participants before enrollment.

\section{Analytical techniques}

Thyroid hormone uptake, total T4 (T4), total T3 (T3), and thyroid stimulating hormone (TSH) were measured using chemiluminescence immunoassays (Access Beckman Coulter, Fullerton CA, USA). A third generation assay was used for TSH determination.

\section{Statistical Analysis}

A previous publication reported a mean ocular protrusion value of $14.82 \mathrm{~mm}$ and standard deviation (SD) of 1.98 mm in healthy Mexican adults [25]. We calculated the sample size considering the hypothesis of at least a $15 \%$ change on proptosis in the groups treated with BRC or PTX. To detect such a difference with an $80 \%$ power and a significance level of 0.05 , each group was designed to comprise 14 patients.

Variables distribution was evaluated using the ShapiroWilk test. Variables are reported using mean \pm SD or median [interquartile range], as appropriate. We evaluated differences in the basal characteristics between groups using one way ANOVA or Kruskal-Wallis test. To evaluate the change in proptosis we used the Wilcoxon signed-rank test. The change in proptosis, CAS, and GO-QOL scores between the three groups was assessed with the Kruskal-Wallis test. Individual comparisons between BRC + MTZ or PTX + MTZ vs MTZ were tested using Mann-Whitney U test. Adverse events reported in all visits between groups were compared using Chi-square or Fisher exact test as appropriate. A p value of less than 0.05 was considered to indicate statistical significance. Statistical analyses were performed with the SPSS version 21. Patients were included in final statistical analysis if they completed evaluations for at least 6 months. The results of the last evaluation were carried forward as the last visit.

\section{Results}

One hundred and thirty seven patients with Graves' disease were evaluated at our Institution between February 


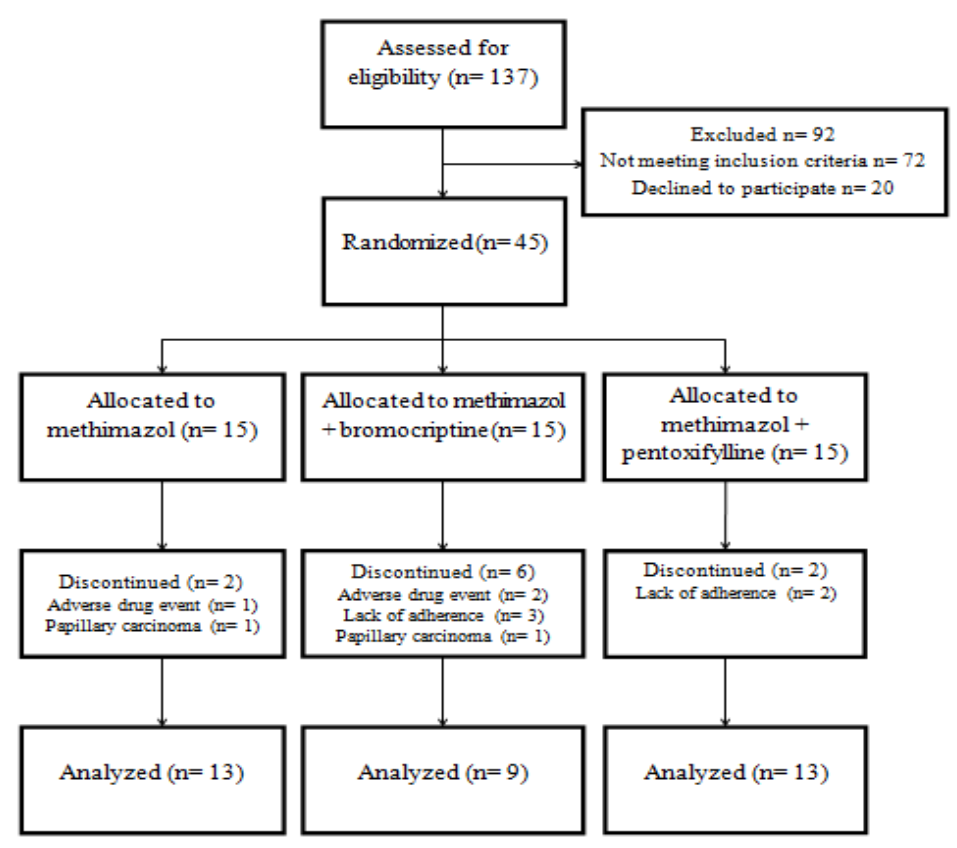

Figure 1: Enrollment, randomization, and follow-up of study patients.

\begin{tabular}{|c|c|c|c|c|}
\hline Variable & $\operatorname{MTZ}(n=13)$ & MTZ + BRC $(n=9)$ & MTZ + PTX $(n=13)$ & $p^{f}$ \\
\hline Age - years & $34.4 \pm 11.2$ & $37.4 \pm 13.5$ & $32.8 \pm 8.7$ & 0.621 \\
\hline Body mass index $-\mathrm{kg} / \mathrm{m}^{2 \mathrm{~b}}$ & $22.6[20.5-26.4]$ & $22.6[20.4-25.2]$ & $23.2[21.3-26.0]$ & 0.864 \\
\hline Heart rate - beats per minute & $95[91-100]$ & $93[80-101]$ & $92[80-99]$ & 0.560 \\
\hline Proptosis OD - mm & 19 [18-20] & $20[18-21]$ & 19 [17.5-19] & 0.562 \\
\hline Proptosis OS - mm & 18 [16-19] & 20 [19-20] & 19 [17.5-19] & 0.143 \\
\hline \multicolumn{5}{|l|}{ Diplopia- number (\%) ${ }^{c}$} \\
\hline Absent & $8(61.5)$ & $6(66.7)$ & $5(38.5)$ & \multirow{3}{*}{0.385} \\
\hline Intermittent & $5(38.5)$ & $1(11.1)$ & $6(46.2)$ & \\
\hline Inconstant & 0 & $2(22.2)$ & $2(15.3)$ & \\
\hline Clinical activity score OD ${ }^{d}$ & $3[3-4]$ & $4[3-4]$ & $4[3-4]$ & 0.664 \\
\hline Clinical activity score OS & $3[3-4]$ & $4[3-4]$ & $4[3-4]$ & 0.420 \\
\hline T3 Uptake - \% ${ }^{\text {e }}$ & $46[43-51]$ & $47[41-50]$ & $0.45[0.41-0.48]$ & 0.544 \\
\hline Total T3 - nmol// ${ }^{\mathrm{e}}$ & $5.63[3.78-8.48]$ & $4.1[3.14-5.74]$ & $4.36[3.47-5.28]$ & 0.252 \\
\hline Total $\mathrm{T} 4-\mathrm{nmol} / \mathrm{l}^{\mathrm{e}}$ & 240 [185.9-283.5] & 254 [191.8-297.9] & $241.7[164.7-280.6]$ & 0.738 \\
\hline $\mathrm{TSH}-\mu \mathrm{U} / \mathrm{ml}^{\mathrm{e}}$ & $0.017 \pm 0.009$ & $0.018 \pm 0.008$ & $0.024 \pm 0.017$ & 0.279 \\
\hline \multicolumn{5}{|c|}{ a Data are expressed as mean \pm SD or median [interquartile range] } \\
\hline \multicolumn{5}{|c|}{${ }^{\mathrm{b}}$ Calculated as the weight in kilograms divided by the square of the height in meters } \\
\hline \multicolumn{5}{|c|}{$\begin{array}{l}{ }^{\mathrm{c}} \text { Evaluated according to the subjective diplopia score as follows intermittent, i.e. diplopia in primary position of gaze, when tired or when first awakening; inconstant, i.e. } \\
\text { diplopia at extremes of gaze; constant, i.e. continuous diplopia in primary or reading position. None individual had constant diplopia }\end{array}$} \\
\hline \multicolumn{5}{|c|}{$\begin{array}{l}\mathrm{d} \text { The clinical activity score (CAS) is the sum of all items present: spontaneous retro bulbar pain, pain on attempted up or down gaze, redness of the eyelids, redness of the } \\
\text { conjunctiva, swelling of the eyelids, inflammation of the caruncle and/or plica, conjunctival edema. A CAS } \geq 3 / 7 \text { indicates active GO }\end{array}$} \\
\hline \multicolumn{5}{|c|}{${ }^{\mathrm{e}}$ Reference values for T3 uptake, total T3, total T4, and TSH are 32-48.4\%, $1.34-2.73 \mathrm{nmol} / \mathrm{l}, 78.3-157.4 \mathrm{nmol} / \mathrm{l}$, and $0.34-5.6 \mu \mathrm{U} / \mathrm{ml}$, respectively } \\
\hline
\end{tabular}

Citataion: J. Gómez-Pérez, Paloma Almeda-Valdés, Daniel Cuevas-Ramos, Miguel A. Gómez-Sámano, et.al. (2017) Favorable Effect of Bromocriptine and Pentoxifylline in Mild Graves Disease Orbitopathy: A Randomized, Open, Clinical Trial. J Endocrinol Diab.4(2):1-8. 
Supplementary Table 1: Baseline characteristics of the participants that completed the study and those not included in the final analysis ${ }^{\mathrm{a}, \mathrm{b}}$

\begin{tabular}{|c|c|c|c|}
\hline Variable & Participants $(n=35)$ & Not included in final analysis $(n=10)$ & $\mathbf{p}$ \\
\hline Age - years & $34.6 \pm 10.8$ & $32.1 \pm 9.2$ & 0.516 \\
\hline Female - number (\%) & $30(85.7)$ & $9(90)$ & 0.598 \\
\hline Body mass index $-\mathrm{kg} / \mathrm{m} 2^{\mathrm{c}}$ & $22.6[21.0-25.7]$ & $23.6[19.8-27.8]$ & 0.799 \\
\hline Systolic blood pressure - mmHg & $120[110-130]$ & $115[110-120]$ & 0.657 \\
\hline Diastolic blood pressure - $\mathrm{mmHg}$ & $70[60-80]$ & $70[70-80]$ & 0.799 \\
\hline Heart rate - beats per minute & $92[81-100]$ & $93.5[80-110]$ & 0.989 \\
\hline Proptosis OD - mm & 19 [17.7-20] & 19 [18.5-20.2] & 0.819 \\
\hline Proptosis OS - mm & 19 [17.5-20] & $19.5[17-21]$ & 0.677 \\
\hline \multicolumn{3}{|l|}{ Diplopia OD - number $(\%)^{\mathrm{d}}$} & 0.735 \\
\hline Absent & $21(60)$ & $7(70)$ & \\
\hline Intermittent & $10(28.6)$ & $3(30)$ & \\
\hline Inconstant & $4(11.2)$ & $2(22.2)$ & \\
\hline \multicolumn{3}{|l|}{ Diplopia- number $(\%)^{d}$} & 0.766 \\
\hline Absent & $19(54.3)$ & $7(70)$ & \\
\hline Intermittent & $12(34.3)$ & $2(20)$ & \\
\hline Inconstant & $3(8.6)$ & $1(10)$ & \\
\hline Clinical activity score OD e & $4[3-4]$ & $3.5[2.5-4]$ & 0.381 \\
\hline Clinical activity score OS & $3[3-4]$ & $4[3-4]$ & 0.819 \\
\hline T3 Uptake - \% ${ }^{\mathrm{f}}$ & $46[42-50]$ & $48[45-51]$ & 0.302 \\
\hline Total T3 - nmol// ${ }^{\mathrm{f}}$ & $4.61[3.59-5.93]$ & $4.41[3.59-8.41]$ & 0.842 \\
\hline Total $\mathrm{T} 4-\mathrm{nmol} / \mathrm{l}^{\mathrm{f}}$ & 241 [189.9-282.2] & 230 [191.6-294.9] & 0.866 \\
\hline $\mathrm{TSH}-\mu \mathrm{U} / \mathrm{ml}^{\mathrm{f}}$ & $0.020 \pm 0.013$ & $0.017 \pm 0.010$ & 0.616 \\
\hline \multicolumn{4}{|c|}{$\begin{array}{l}\text { d Evaluated according to the subjective diplopia score as follows intermittent, i.e. diplopia in primary position of gaze, when tired or } \\
\text { when first awakening; inconstant, i.e. diplopia at extremes of gaze; constant, i.e. continuous diplopia in primary or reading position. } \\
\text { None individual had constant diplopia } \\
\text { e The clinical activity score (CAS) is the sum of all items present: spontaneous retrobulbar pain, pain on attempted up or down gaze, } \\
\text { redness of the eyelids, redness of the conjunctiva, swelling of the eyelids, inflammation of the caruncle and/or plica, conjunctival } \\
\text { edema. A CAS } \geq 3 / 7 \text { indicates active GO }\end{array}$} \\
\hline
\end{tabular}


2009 and July 2012, 65 patients who fulfilled the inclusion criteria were invited to participate, and 45 were randomly assigned to receive MTZ, MTZ + BRC, or MTZ + PTX treatment. Of the randomized 45 patients, 10 left the study before 6-months (Figure 1). These patients were not included in the final analysis; however, their baseline characteristics were not different from the patients that completed the study (supplementary Table 1). Baseline characteristics of included patients were not different between groups (Table 1). During follow-up all patients achieved euthyroidism with MTZ treatment and levothyroxine replacement was added as needed.

\section{Change in proptosis}

Proptosis decreased significantly with BRC therapy. In OD from 20 [18-21] to 18 [16-18] $\mathrm{mm}(\mathrm{p}=0.006)$, and in OS from 20 [19-20] to 18 [16-18] $\mathrm{mm}(\mathrm{p}=0.006)$. Proptosis also decreased with PTX treatment; however, this was significant only in OS. In OD decreased from 19 [17.5-19] to 16.5 [16-19] $\mathrm{mm}(\mathrm{p}=$ 0.120 ) and in OS from 19 [17.5-19] to 17 [16-20] mm ( $p=0.025)$. In contrast, the group treated only with MTZ showed no change in proptosis. In OD from 19 [18-20] to 19 [16.5-19] mm ( $p=0.173)$, and in OS from 18 [16-19] to 18 [16-20] mm (p=0.942). These results are depicted in figure 2 .

The change in proptosis, calculated as the baseline proptosis - final proptosis, was compared between groups. As shown in Table 2, proptosis decreased significantly in both eyes with BRC. The change in proptosis with PTX was significant only in OS.

Table 2: Change in proptosis in each treatment group ${ }^{\text {a }}$

\begin{tabular}{|c|c|c|c|c|c|}
\hline \multirow{2}{*}{$\begin{array}{c}\text { Proptosis } \\
\text { change } \\
\text { (mm) }\end{array}$} & \multirow[b]{2}{*}{$\begin{array}{c}\text { MTZ } \\
(n=13)\end{array}$} & \multirow{2}{*}{$\begin{array}{c}\text { MTZ } \\
+ \text { BRC } \\
(n=9)\end{array}$} & \multirow{2}{*}{$\begin{array}{c}\text { MTZ } \\
+ \text { PTX } \\
(n=13)\end{array}$} & \multicolumn{2}{|c|}{$\mathrm{p} \dagger$} \\
\hline & & & & MTZ vs. BRC & $\begin{array}{c}\text { MTZ vs. } \\
\text { PTX }\end{array}$ \\
\hline OD & $0[0-2]$ & $2[2-2]$ & $1[0-3]$ & 0.014 & 0.343 \\
\hline OS & $0[-1-1]$ & $2[2-2]$ & 1 [1 - 2] & $<0.001$ & 0.015 \\
\hline
\end{tabular}

* Values are medians [interquartile range]

${ }^{\dagger} \mathrm{P}$ values obtained from individual comparisons between BRC and PTX with MTZ treatments using Mann-Whitney U test

\section{Change in clinical activity score and quality of life}

The CAS showed similar reduction in all groups $(\mathrm{p}=$ 0.693 and $p=0.188$ for OD and OS, respectively). Four subjects did not complete the final GO-QOL questionnaire; therefore this analysis includes 31 subjects (12 in the MTZ, 9 in the MTZ + BRC, and 10 in the MTZ + PTX groups). At the beginning of the study, the scores in the visual functioning and appearance subscales were not different between groups $(\mathrm{p}=0.769$ and $\mathrm{p}=0.502$, respectively). At the end of the study, an improvement in both the visual functioning and appearance scores was documented. The median CAS and GO-QOL scores at the beginning and at the end of the study are summarized on Table 3.
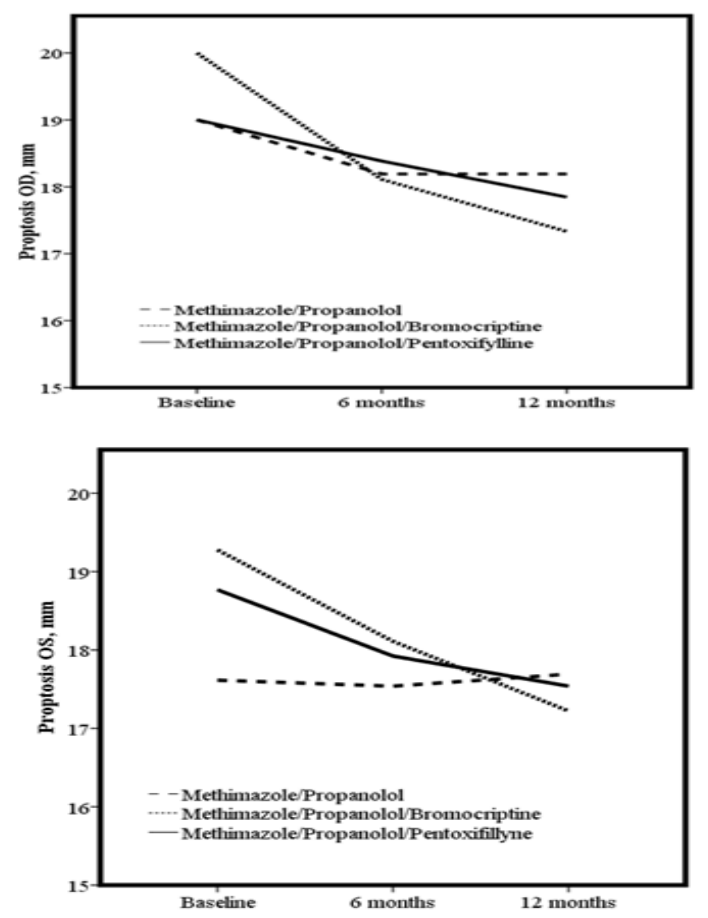

Figure 2: Change in proptosis. BRC and PTX decreased proptosis in both eyes. The decrease with PTX was significant only in the OS. Lines represents the median proptosis value at baseline, six, and 12 months. A. Proptosis in OD at the beginning, six months, and at the end of the study B. Proptosis in OS at the beginning, six months, and at the end of the study.

\section{Adverse events}

An increased frequency of diarrhea was documented in the PTX + MTZ group $(\mathrm{p}=0.026)$. The frequency of other adverse events was similar between groups (Table 4). Three patients left the study before 6 months due to adverse events. One patient presented a skin reaction in the MTZ group, one patient in MTZ+BRC group developed febrile neutropenia, and one more in the MTZ+BRC group suspended treatment because nausea and vomit.

\section{Discussion}

In patients with mild GO, treatment with BRC in addition to MTZ resulted in a significant reduction in proptosis. The benefit was also seen with PTX plus MTZ; however, it was only significant on OS. This favorable outcome was associated with an improvement in the CAS and in the quality of life. The proptosis did not change in patients treated with MTZ alone.

The pathophysiology of proptosis involves an increase in glycosaminoglycan production, adipose tissue hypertrophy, and edema of orbital tissues [26]. The fibroblasts are key cells involved in the development of GO [27], they can differentiate into mature adipocytes [28] with an increased expression of the thyroid stimulating hormone receptor [29]. Other fibroblasts are responsive to cytokines such as the transforming growth factor beta (TGF- $\beta$ ) and can differentiate into myofibroblasts, participating in processes such as inflammation, repair, and fibrosis [30, 
Table 3: Clinical activity score (CAS) and Graves' Orbitopathy Quality Of Life (QOL-GO) questionnaire scores at the beginning and end of the study a

\begin{tabular}{|l|l|l|l|l|l|l}
\hline & MTZ $(n=13)$ & MTZ + BRC (n= 9) & MTZ + PXF (n= 13) & \multicolumn{2}{|c|}{$P^{f}$} \\
\hline Baseline CAS OD ${ }^{\mathrm{f}}$ & $3[3-4]$ & $4[3-4]$ & $4[3-4]$ & 0.393 & 0.65 \\
\hline 6-month CAS OD & $2[1-2]$ & $1[0-2]$ & $0[0-1]$ & 0.262 & 0.034 \\
\hline Final CAS OD & $1[0-2]$ & $0[0-1]$ & $0[0-1]$ & 0.556 & 0.418 \\
\hline Baseline CAS OI & $3[3-4]$ & $4[3-4]$ & $4[3-4]$ & 0.292 & 0.336 \\
\hline 6-month CAS OI & $1[1-2]$ & $1[0-2]$ & $1[0-2]$ & 0.965 & 0.287 \\
\hline Final CAS ${ }^{\text {d }}$ & $1[0-2]$ & $0[0-1]$ & $1[0-1]$ & 0.357 & 0.614 \\
\hline Baseline visual functioning scored & $75[43.7-96.9]$ & $56.2[50.0-75.0]$ & $73.2[43.7-78.6]$ & 0.651 & 0.497 \\
\hline Final visual functioning score & $96.9[87.5-100]$ & $100[83.3-100]$ & $100[93.7-100]$ & 0.862 & 0.832 \\
\hline Baseline appearance score & $71.9[43.7-84.4]$ & $75.0[68.7-93.7]$ & $75.0[56.2-87.5]$ & 0.382 & 0.314 \\
\hline Final appearance score & $93.7[81.2-96.9]$ & $100[93.7-100]$ & $96.9[93.7-100]$ & 0.148 & 0.228
\end{tabular}

a Values are medians [interquartile range].

${ }^{\mathrm{b}} \mathrm{p}$ values obtained from individual comparisons between BRC or PTX with MTZ treatments using Mann-Whitney U test.

' The clinical activity score (CAS) is the sum of all items present: spontaneous retro bulbar pain, pain on attempted up or down gaze, redness of the eyelids, redness of the conjunctiva, swelling of the eyelids, inflammation of the caruncle and/or plica, conjunctival edema. A CAS $\geq 3 / 7$ indicates active GO.

d The final score ranges from a minimum of 0 (full limitation) to 100 (no limitation). An increase in the score indicates improvement and a decrease indicates worsening.

\begin{tabular}{|c|c|c|c|c|}
\hline Event & MTZ (n= 13) & MTZ + BRC $(n=9)$ & MTZ + PTX $(n=13)$ & $\mathbf{P}^{\mathbf{b}}$ \\
\hline Orthostatic hypotension & 0 & $3(2)$ & $4(3)$ & 0.12 \\
\hline Epistaxis & $5(2)$ & $2(2)$ & $7(2)$ & 0.497 \\
\hline Skin reaction & $3(2)$ & $4(3)$ & $4(4)$ & 0.67 \\
\hline Nausea and/or vomit & $9(5)$ & $8(5)$ & $13(6)$ & 0.67 \\
\hline Diarrhea & $5(4)$ & $2(1)$ & $13(6)$ & 0.026 \\
\hline Edema & $7(4)$ & $2(1)$ & $10(6)$ & 0.212 \\
\hline Bronchospasm & 0 & $2(1)$ & 1 & 0.211 \\
\hline Pharyngitis & 1 & 1 & $2(2)$ & 0.843 \\
\hline Fever & 1 & 1 & 1 & 0.955 \\
\hline Anorexia & 1 & $2(1)$ & 1 & 0.535 \\
\hline Pruritus & $2(2)$ & 1 & $3(2)$ & 0.783 \\
\hline Abdominal pain & $12(5)$ & $10(4)$ & $12(4)$ & 0.874 \\
\hline Arthralgia & 1 & 1 & 0 & 0.523 \\
\hline Mild neutropenia & 1 & 0 & 0 & 0.428 \\
\hline Somnolence & $13(5)$ & $14(7)$ & $16(7)$ & 0.467 \\
\hline Headache & $9(4)$ & $11(6)$ & $12(6)$ & 0.407 \\
\hline
\end{tabular}

Citataion: J. Gómez-Pérez, Paloma Almeda-Valdés, Daniel Cuevas-Ramos, Miguel A. Gómez-Sámano, et.al. (2017) Favorable Effect of Bromocriptine and Pentoxifylline in Mild Graves Disease Orbitopathy: A Randomized, Open, Clinical Trial. J Endocrinol Diab.4(2):1-8. 
31]. In addition, angiogenesis could also play a role in the pathogenesis of GO [32, 33]. BRC and PTX can decrease inflammation by several mechanisms, and these can be beneficial in patients with proptosis due to GO.

$\mathrm{BRC}$ is a dopamine 2 receptor agonist. It has direct effects on immune cells that seem to be independent of PRL levels [34]. However, there is evidence of an association between PRL levels and autoimmune disease. Reduction of PRL causes a decline of interferon gamma and IL-2 secretion by Th1 lymphocytes [20]. A possible mechanism explaining the improvement in GO is its anti-proliferative effect on T lymphocytes by blocking IL-2 production $[13,35]$. Furthermore, BRC suppresses human B lymphocyte function along with immunoglobulin production [35]. Finally, an additional effect of dopaminergic agonists is inhibition of the vascular endothelial growth factor (VEGF) [36], which can be an additional mechanism involved in clinical improvement in proptosis in patients with mild GO of recent onset. Some reports have found increased serum levels of VEGF in individuals with GO in comparison with controls [32].

The particular effect of PTX in GO is the inhibition of tumor necrosis factor alpha synthesis [37]. Also, using fibroblast cell cultures and animal models, an anti-fibrotic effect has been reported [38]. These two mechanisms may contribute to the favorable effect in GO.

It is well known that MTZ improves the CAS [3]. Since the three groups in our study received MTZ, it is not unexpected the lack of statistical significance between CAS score between the three groups.

Our results are consistent with previous case reports and small case series describing a possible beneficial effect of BRC in individuals with GO $[13,15]$.

It is important to emphasize that we treated patients with less than one year of GO onset. This fact decreases the possibility of development of fibrosis that could limit the improvement in proptosis.

This study has limitations that should be mentioned. First, we include a small number of patients. However, participants were carefully selected and followed, and sufficient statistical power was achieved to detect significant results with clinical relevance. Nevertheless, it can be also possible that lack of study power was the reason to find a non-significant effect of PTX treatment on proptosis on the OD. The results of this study cannot be generalized to patients with moderate or severe GO in whom other interventions are required. Finally, although we postulate potential mechanisms for the observed improvement in the proptosis, the confirmation of these mechanisms will be the subject of future studies.

\section{Conclusion}

In summary the results of this study indicate that BRC and PTX treatment added to MTZ improve proptosis, CAS and quality of life in individuals mild GO of recent onset.

Disclosure statement: The authors declare that no financial or other conflicts of interest exist in relation to the content of the article.

\section{References}

1. Bartley GB. The epidemiologic characteristics and clinical course of ophthalmopathy associated with autoimmune thyroid disease in Olmsted County, Minnesota. Trans Am Ophthalmol Soc. 1994;92:477-588.

2. Lazarus JH. Epidemiology of Graves' orbitopathy (GO) and relationship with thyroid disease. Best Pract Res Clin Endocrinol Metab. 2012;26(3):273-279.

3. Bartalena L, Baldeschi L, Dickinson AJ, Eckstein A, Taylor PK, Marcocci C,et al. Consensus statement of the European group on Graves' orbitopathy (EUGOGO) on management of Graves' orbitopathy. Thyroid. 2008;18(3):333-346. Doi: 10.1089/thy.2007.0315

4. Bartalena L. Graves' orbitopathy: imperfect treatments for a rare disease. Eur Thyroid J. 2013;2(4):259-269. Doi: 10.1159/000356042

5. Gerding MN, Terwee CB, Dekker FW, Koornneef L, Prummel MF, Wiersinga WM. Quality of life in patients with Graves' ophthalmopathy is markedly decreased: measurement by the medical outcomes study instrument. Thyroid. 1997;7(6):885-889. Doi: 10.1089/thy.1997.7.885

6. Perros P, Crombie AL, Kendall-Taylor P. Natural history of thyroid associated ophthalmopathy. Clin Endocrinol (Oxf). 1995;42(1):45-50.

7. Wemeau JL, Caron P, Beckers A, Rohmer V, Orgiazzi J, Borson-Chazot F,et al. Octreotide (long-acting release formulation) treatment in patients with graves' orbitopathy: clinical results of a four-month, randomized, placebo-controlled, double-blind study. J Clin Endocrinol Metab. 2005;90(2):841-848. Doi: 10.1210/jc.2004-1334

8. Stamato FJ, Maciel RM, Manso PG, Wolosker AM, Paiva ER, Lopes AC et al. Colchicine in the treatment of the inflammatory phase of Graves' ophthalmopathy: a prospective and randomized trial with prednisone. Arq Bras Oftalmol. 2006;69(6):811-816.

9. Bouzas EA, Karadimas P, Mastorakos G, Koutras DA. Antioxidant agents in the treatment of Graves' ophthalmopathy. Am J Ophthalmol. 2000;129(5):618-622.

10. Bloise W, Mimura LY, Moura J, Nicolau W. Treatment of mild to moderate Graves' ophthalmopathy with sodium diclofenac: a pilot study. Arq Bras Endocrinol Metabol. 2011;55(9):692-695.

11. Marcocci C, Kahaly GJ, Krassas GE, Bartalena L, Prummel M, Stahl M, et al. Selenium and the course of mild Graves' orbitopathy. N Engl J Med. 2011;364(20):1920-1931. Doi: 10.1056/NEJMoa1012985

12. Finamor FE, Martins JR, Nakanami D, Paiva ER, Manso PG, Furlanetto RP, et al. Pentoxifylline (PTX)--an alternative treatment in Graves' ophthalmopathy (inactive phase): assessment by a disease specific quality of life questionnaire and by exophthalmometry in a prospective randomized trial. Eur J Ophthalmol. 2004;14(4):277-283.

13. Kolodziej-Maciejewska H, Reterski Z. Positive effect of bromocriptine treatment in Graves disease orbitopathy. Exp Clin Endocrinol. 1985;86(2):241-242.

14. Zinkevich IV, Kazeev KN, Poliak AI, Zhuravlev VS. [Dynamics of clinicoimmunologic indices in patients with endocrine ophthalmopathy undergoing treatment with the preparation parlodel]. Probl Endokrinol (Mosk). 1988;34(6):6-9.

15. Lopatynsky MO, Krohel GB. Bromocriptine therapy for thyroid ophthalmopathy. Am J Ophthalmol. 1989;107(6):680-681.

16. Abraham P, Avenell A, Watson WA, Park CM, Bevan JS. Antithyroid drug regimen for treating Graves' hyperthyroidism. Cochrane Database Syst Rev. 2005;18(2):CD003420. Doi: 10.1002/14651858.CD003420.pub3 
17. Xu D, Lin L, Lin X, Huang Z, Lei Z. Immunoregulation of autocrine prolactin: suppressing the expression of costimulatory molecules and cytokines in T lymphocytes by prolactin receptor knockdown. Cell Immunol. 2010;263(1):71-78. Doi: 10.1016/j.cellimm.2010.02.018

18. Ignacak A, Kasztelnik M, Sliwa T, Korbut RA, Rajda K, Guzik TJ. Prolactin--not only lactotrophin. A "new" view of the "old" hormone. J Physiol Pharmacol. 2012;63(5):435-443.

19. Shelly S, Boaz M, Orbach H. Prolactin and autoimmunity. Autoimmun Rev. 2012;11(6-7):A465-470. Doi: 10.1016/j.autrev.2011.11.009

20.De Bellis A, Bizzarro A, Pivonello R, Lombardi G, Bellastella A. Prolactin and autoimmunity. Pituitary. 2005;8(1):25-30. Doi: 10.1007/ s11102-005-5082-5

21. Vera-Lastra O, Jara LJ, Espinoza LR. Prolactin and autoimmunity. Autoimmun Rev. 2002;1(6):360-364.

22. Chikanza IC. Prolactin and neuroimmunomodulation: in vitro and in vivo observations. Ann N Y Acad Sci. 1999;876:119-130.

23. Mourits MP, Prummel MF, Wiersinga WM, Koornneef L. Clinical activity score as a guide in the management of patients with Graves' ophthalmopathy. Clin Endocrinol (Oxf). 1997;47(1):9-14.

24. Terwee CB, Dekker FW, Mourits MP, Gerding MN, Baldeschi L, Kalmann R. Interpretation and validity of changes in scores on the Graves' ophthalmopathy quality of life questionnaire (GO-QOL) after different treatments. Clin Endocrinol (Oxf). 2001;54(3):391-398.

25. Bolanos Gil de Montes F, Perez Resinas FM, Rodriguez Garcia M, González Ortiz M. Exophthalmometry in Mexican adults. Rev Invest Clin. 1999;51(6):341-343.

26. Perros P, Kendall-Taylor P. Thyroid-associated ophthalmopathy: pathogenesis and clinical management. Baillieres Clin Endocrinol Metab. 1995;9(1):115-135.

27. Prabhakar BS, Bahn RS, Smith TJ. Current perspective on the pathogenesis of Graves' disease and ophthalmopathy. Endocr Rev. 2003;24(6):802-835.

28. Sorisky A, Pardasani D, Gagnon A, Smith TJ.et al. Evidence of adipocyte differentiation in human orbital fibroblasts in primary culture. J Clin Endocrinol Metab. 1996;81(9):3428-3431.

29. Valyasevi RW, Erickson DZ, Harteneck DA, Dutton CM, Heufelder AE, Jyonouchi SC, et al. Differentiation of human orbital preadipocyte fi- broblasts induces expression of functional thyrotropin receptor. J Clin Endocrinol Metab. 1999;84(7):2557-2562.

30. Smith TJ, Koumas L, Gagnon A, Bell A, Sempowski GD, Phipps RP. Orbital fibroblast heterogeneity may determine the clinical presentation of thyroid-associated ophthalmopathy. J Clin Endocrinol Metab. 2002;87(1):385-392.

31. Pawlowski P, Reszec J, Eckstein A, Kristian Johnson, Andrzej Grzybowski, et al.. Markers of inflammation and fibrosis in the orbital fat/ connective tissue of patients with Graves' orbitopathy: clinical implications. Mediators Inflamm. 2014;2014:412158.

32. Nowak M, Marek B, Karpe J, Kos-Kudla B, Sieminska L, Kajdaniuk D. Serum Concentration of VEGF and PDGF-AA in Patients with Active Thyroid Orbitopathy before and after Immunosuppressive Therapy. Exp Clin Endocrinol Diabetes. 2014;122(10):582-586.

33. Kajdaniuk D, Marek B, Niedziolka-Zielonka D, Wanda Foltyn, Mariusz Nowak, Lucyna Siemińska, et al. Transforming Growth Factor beta1 (TGFbeta1) and Vascular Endothelial Growth Factor (VEGF) in the blood of healthy people and patients with Graves' orbitopathy - a new mechanism of glucocorticoids action? Endokrynol Pol. 2014;65(5):348-356.

34. Dorshkind K, Horseman ND. The roles of prolactin, growth hormone, insulin-like growth factor-I, and thyroid hormones in lymphocyte development and function: insights from genetic models of hormone and hormone receptor deficiency. Endocr Rev. 2000;21(3):292-312.

35. Morkawa K, Oseko F, Morikawa S. Immunosuppressive property of bromocriptine on human B lymphocyte function in vitro. Clin Exp Immunol. 1993;93(2):200-205.

36. Gagliano T, Filieri C, Minoia M, Buratto M, Tagliati F, Ambrosio MR. Cabergoline reduces cell viability in non functioning pituitary adenomas by inhibiting vascular endothelial growth factor secretion. Pituitary. 2013;16(1):91-100.

37. Balazs C, Kiss E, Vamos A, Molnar I, Farid NR. Beneficial effect of pentoxifylline on thyroid associated ophthalmopathy (TAO)*: a pilot study. J Clin Endocrinol Metab. 1997;82(6):1999-2002.

38. Windmeier C, Gressner AM. Pharmacological aspects of pentoxifylline with emphasis on its inhibitory actions on hepatic fibrogenesis. Gen Pharmacol. 1997;29(2):181-196. 